\title{
YAO: a generator of parallel code for variational data assimilation applications
}

\author{
Luigi Nardi* ${ }^{*}$, Fouad Badran ${ }^{\dagger}$, Pierre Fortin ${ }^{\ddagger}$ and Sylvie Thiria* \\ * LOCEAN, Laboratoire d'Océanographie et du Climat: Expérimentations et approches numériques. \\ UMR 7159 CNRS / IRD / Université Pierre et Marie Curie / MNHN. \\ Institut Pierre Simon Laplace. 4, place Jussieu Paris 75005, France. \\ $\dagger$ CEDRIC, Centre d'Etude et De Recherche en Informatique du CNAM. EA 1395, \\ 292 rue St Martin Paris 75003, France. \\ $\ddagger$ UPMC Univ Paris 06 and CNRS UMR 7606, LIP6, 4 place Jussieu, F-75252, Paris cedex 05, France
}

\begin{abstract}
Variational data assimilation consists in estimating control parameters of a numerical model in order to minimize the misfit between the forecast values and the actual observations. The YAO framework is a code generator that facilitates, especially for the adjoint model, the writing and the generation of a variational data assimilation program for a given numerical application. In this paper we present how the modular graph specific to YAO enables the automatic and efficient parallelization of the generated code with OpenMP on shared memory architectures. Thanks to this modular graph we are also able to completely avoid the data race conditions (write/write conflicts). Performance tests with actual applications demonstrates good speedups on a multicore CPU.
\end{abstract}

Keywords: data assimilation; automatic parallelization; shared memory architectures; OpenMP; dependence graph; numerical model; adjoint model

\section{INTRODUCTION}

Numerical models are widely used for studying physical phenomena. Most of the time, the model is used to forecast or analyze the evolution of the phenomenon. Since the model is imperfect, discrepancy between its forecast values and actual observations may be important due to model parametrization, numerical discretization, uncertainty on the initial conditions and boundary conditions. A new method, the so-called data assimilation [1], which uses both the numerical model of the phenomenon and the inverse problem method has been introduced to reduce this discrepancy. Data assimilation uses actual observations to constrain the control parameters (initial conditions, model parameters, ...) in order to force the numerical model (thereafter also referred to as the direct model) to reproduce the desired behavior. In variational data assimilation methods [2], this task is done by minimizing, with respect to control parameters, a cost function $J$ which measures the misfit between the direct numerical model outputs and the observations. The minimization is done by the use of a gradient method, which requires calculating the gradient of $J$ as a function of the control parameters. The gradient computation requires the product of the transpose Jacobian matrix of the direct numerical model with the derivative vector of $J$ defined at the observation points. This product is also called adjoint model. Since the direct numerical model is usually very complex, the implementation of the programming code which represents the adjoint model is very complicated.

The YAO framework already presented in [3], [4] is a code generator dedicated to variational data assimilation. With YAO the user defines, using specific directives and $\mathrm{C}$ programming, the type of discretization and the specification of the numerical model. It then generates automatically the numerical and the adjoint model codes via $\mathrm{C}++$ object-oriented programming. YAO has already been used with success on several actual applications in oceanography: Shallow-water [3], [4], Marine acoustics [5], [6], Ocean color [7], PISCES [8], GYRE configuration of $N E M O$ [9].

The YAO formalism is based on a modular graph, which is similar to those used in automatic parallelization of nested loops. In this well-developed research field several concepts and algorithms have been introduced, which allow the analysis of nested loops, their decomposition and fusion [10], [11], [12]. The decomposition obtained is well suited to a multithread parallelization on shared memory architectures where no communications are required. In this paper, we show how the YAO modular graph enables us to integrate and adapt these algorithms in order to identify the available parallelism, and to allow the automatic generation of parallel code with YAO while completely avoiding the data race conditions (write/write conflicts). With the OpenMP directives, it is then possible to generate a multi-threaded parallel code that runs efficiently on shared memory architectures. This is an important improvement over the previous version of YAO [3] which can generate only sequential code. A large community in geophysics may thus automatically and transparently exploit decades of research in automatic parallelization and benefit from important speedups in computation times on multicore architectures without any additional effort and without any knowledge in parallel programming.

For the automatic generation of parallel code, the development of algorithms specific to YAO is necessary. Indeed, the software tools for automatic parallelization with OpenMP directives have specific constraints related to their design, and can therefore currently not be integrated in the YAO generator. For example, the CAPO toolkit [13] supports only Fortran 
and relies on user interaction to improve the parallelization process. The Gaspard2 framework [14] enables automatic OpenMP code generation, but the available parallelism must be first specified by the user in an UML model. The PLuTo tool [15] can efficiently parallelize nested loops while taking into account, via tiling, data locality on multicore architectures with complex hierarchical memory. However PLuTo does not currently support object-oriented programming for input source codes and it has specific limitations (only SCoP programs with pure function calls, no dynamic branch conditions) that also prevents a direct integration in YAO. Finally and most importantly, as detailed further there are data race conditions (write/write conflicts) in the generated code. These data race conditions prevent any automatic parallelization from such tools according to their own data-dependency analysis. To our knowledge, none of these tools can automatically insert (for example) OpenMP atomic directives to avoid these race conditions and thus enable the parallelization. We here show how to efficiently accomplish this in YAO thanks to its modular graph.

Adapting state-of-the-art algorithms to YAO while relying on its modular graph has also several advantages. First, there is no constraint on the application code written by the user. Second, a high-level dependency graph is directly provided by the modular graph which enables to avoid the data-dependency analysis, to naturally obtain a coarse grain parallelism, and to possibly scale on real-life applications with thousands of statements.

In the following, we will first give a brief overview of the YAO framework in section II. Then, in section III we will show how the YAO modular graph can be used to automatically and efficiently parallelize the generated code on shared memory architectures. Performance results for two actual YAO applications on a multicore CPU are detailed in section IV. Finally, in section V concluding remarks are presented and future work discussed.

\section{YAO PRESENTATION}

\section{A. The modular graph}

We present here the concept of modular graph, which is fundamental in YAO, as well as the forward and backward procedures: more details can be found in [3], [4]. We first define the following terms.

- A module is an entity of computation; it receives inputs from other modules or from an external context ${ }^{1}$ and it transmits outputs to other modules or to an external context.

- A connection is a transmission of data from a module to another or between a module and an external context.

- A modular graph is a data flow graph composed by a set of several interconnected modules; it summarizes the sequential order of the computations.

\footnotetext{
${ }^{1}$ An external context is an entity which initializes and retrieves the computation of certain modules.
}

In order to perform data assimilation, at each time step a modular graph is traversed by the forward procedure and then by the backward procedure.

1) The forward procedure: the input data set of a module $F_{p}$ is a vector denoted $\mathbf{x}_{p}$ and its output data set is a vector denoted $\mathbf{y}_{p}\left(\mathbf{y}_{p}=F_{p}\left(\mathbf{x}_{p}\right)\right)$. As a consequence, a module $F_{p}$ can be executed only if its input vector $\mathbf{x}_{p}$ has already been processed, which implies that all its predecessor modules have been executed beforehand. Thus there are only flow dependencies [10] between modules. Since we suppose that the modular graph is acyclic, it is then possible to find a module ordering, i.e. a topological order, which allows us to correctly propagate the calculation through the graph. If we denote by $\mathbf{x}$ the vector corresponding to all the graph input data, provided by the external context, the forward procedure enables to calculate the vector $\mathbf{y}$ corresponding to all the graph output values. The modular graph defines an overall function $\Gamma$ and makes it possible to compute $\mathbf{y}=\Gamma(\mathbf{x})$. The function $\Gamma$ has a physical meaning: it represents a direct numerical model $M$, with respect to the YAO formalism. The forward procedure allows us to compute the outputs of the numerical model according to its inputs. The incoming connections from the external context of $\Gamma$ could be, for example, initializations or boundary conditions. Outgoing connections transmit their values to compute, as an example, a cost function.

2) The backward procedure: this procedure enables the computation of the adjoint of the cost function $J$ with respect to the control parameters. We suppose that for each module $F_{p}$, with an input vector $\mathbf{x}_{p}$ and receiving in its output data points a "perturbation" vector $\mathbf{d} \mathbf{y}_{p}$, we can compute the matrix product $\mathbf{d} \mathbf{x}_{p}=\mathbf{F}_{p}^{T} \mathbf{d} \mathbf{y}_{p}, \mathbf{F}_{p}^{T}$ being the transposed Jacobian matrix of the module $F_{p}$ calculated at point $\mathbf{x}_{p}$. It is possible [4] to compute the gradient of $J$ with respect to the control parameters by traversing the modular graph in a reverse topological order and executing local computations on each module in order to compute $\mathbf{d x} \mathbf{x}_{p}$. It has been shown [3], [4] that this reversed traversal leads to a back propagation on the modular graph characterised by additions (accumulations) of several local computations. Each of these additions is computed in an intermediate step and then back propagated. Thus there are flow and output dependencies [10].

3) YAO formalism: running simulations or data assimilations using an operational numerical model $M$ requires the definition of a modular graph representing the sequence of the computations. A numerical model operates on a discrete grid, where the physical process is computed at each grid point $I$ and at each time step $t$. As the phenomenon under study is quite the same at each grid point, only the modular subgraph representing a grid point is needed. YAO obtains $\Gamma$ by duplicating this subgraph for each $I$ and $t$.

In the YAO formalism, the user must define a set of basic functions $\left\{F_{1}, F_{2}, \ldots F_{k}\right\}$ which has to be applied to each grid point $I$ and at each time step $t$. The user has to define also the dependencies between these functions. From this information, YAO generates the overall modular graph $\Gamma$. The modules of the modular subgraph $\Gamma_{I, t}$ are denoted by $F_{p}(I, t)$, where $I$ 


\begin{tabular}{|c|c|c|c|}
\hline ctin $F_{1}$ & from $F_{1}$ & $i$ & $j-1$ \\
\hline ctin $F_{2}$ & from $F_{1}$ & i & $j+1$ \\
\hline $\operatorname{ctin} F_{2}$ & from $F_{3}$ & $i-1$ & $j+1$ \\
\hline $\operatorname{ctin} F_{2}$ & from $F_{3}$ & i & j \\
\hline ctin $F_{2}$ & from $F_{4}$ & $i+1$ & j \\
\hline $\ln F_{3}$ & from $F_{1}$ & $i-1$ & j \\
\hline n $F_{4}$ & from $F_{3}$ & i & j \\
\hline$F_{4}$ & from $F_{2}$ & $i$ & $j+1$ \\
\hline
\end{tabular}

Fig. 1. (a) Part of the specification language used by the user with 2D space modules. The second ctin directive specifies the connection from $F_{1}$ at point $(\mathrm{i}, \mathrm{j}+1, \mathrm{t})$ to $F_{2}$ at point $(\mathrm{i}, \mathrm{j}, \mathrm{t})$. (b) The order directives indicate the ordering in which we compute the functions $F_{p}$ and the ordering in which we traverse the grid.

represents a grid point (1D, 2D or 3D), $t$ is a time step and $F_{p}$ a basic function. Thus, a module is the computation of the function $F_{p}$ at grid point $I$ and at time $t$. We denote by $i$ (respectively $j$ and $k$ ) the indices of the first axis (resp. the second and third axis). An edge from a source module $F_{s}\left(I^{\prime}, t^{\prime}\right)$ to a destination module $F_{d}(I, t)$ corresponds to a data transmission from $F_{s}\left(I^{\prime}, t^{\prime}\right)$ to $F_{d}(I, t)$ ( $s$ may be equal to $d$ ).

The modular graph is similar to the Expanded Dependence Graph (EDG) used for the parallelism detection in nested loops [10]. The main difference with the modular graph being that in the EDG the nodes represent one operation (the instance of a statement) while the nodes of the modular graph are a set of operations (the instance of a function composed by a set of statements) represented by the module $F_{p}(I, t)$. Thus, the granularity of the nodes differs. In practice the dimension of a YAO basic function depends on the application and on the user design. In general a YAO module has some dozens of statements but in particular cases it may be very much larger.

\section{B. User specifications and code generation}

This section presents two YAO directives, ctin and order, on which relies the YAO automatic code generation. These directives generate nested loops, which allow us to traverse the modules $F_{p}(I, t)$ in the correct ordering.

1) order and ctin directives: the ctin directive has the following syntax "ctin from $F_{s}$ to $F_{d}$ list of coordinates". Such a directive represents one edge (or connection) of the modular graph which is then automatically replicated by YAO on space and time. list of coordinates represents, for a generic point $I$ and time step $t$ of the destination module $F_{d}$, the point $I^{\prime}$ and the time $t^{\prime}$ of the source module $F_{s}$ (with $t \geq t^{\prime}$ ). The difference $I^{\prime}-I$ represents the distance vector of the source module with respect to the destination module; we denote its coordinates by $d_{i}, d_{j}$ or $d_{k}$. The user has to specify in the list of coordinates the distance vector and $d_{t}=t^{\prime}-t$ as a function of the generic point $I$ of the destination module, which is the same in all connections. Fig. 1a gives an example of ctin directives.

Every ctin directive generates an edge from $F_{s}$ to $F_{d}$ labeled

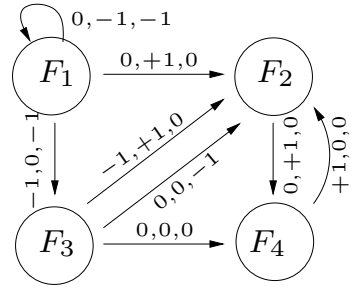

Fig. 2. RDG issued by the ctin directives of Fig. 1a.

by the distance vector $I^{\prime}-I$ and $t^{\prime}-t$. The resulting graph is a directed multigraph ${ }^{2}$ which represents all dependencies between basic functions. This multigraph corresponds to the Reduced Dependence Graph (RDG) [10] used for the automatic generation of parallelism in nested loops ${ }^{3}$. Fig. 2 presents the RDG of the former example. Since the space dimension is two, the edges are labeled by $\left(d_{i}, d_{j}, d_{t}\right)$ which indicates that the destination module, at time $\mathrm{t}$ and at point $(i, j)$, takes its inputs from the source module at time $t+d_{t}$ and point $\left(i+d_{i}, j+d_{j}\right)$ with $d_{i}, d_{j} \in \mathbb{Z}$ and $d_{t} \in \mathbb{Z}_{<0}$.

The YAO order directive allows the user to define a traversal of the modular graph following a topological order. This directive allows to visit all the grid points of the space, and permits the generation of nested loops. The user specifies one order directive for each dimension of the space. Thus, a program generated by YAO, contains an outermost loop, that represents the time, within which the user defines, thanks to the order directives, the different loops that allows the traversal of the space for each time step. In general, we have several ways to traverse a space. In the order directive, YAl (YAO Afterward axis 1 ) means that we are managing the $i$ loop and we go along this axis in an ascendant way. YA2 means the same but for the $j$ axis, whereas $Y B 1$ (YAO Backward axis 1 ) means that we go along the $i$ axis in a descendant way. Fig. $1 \mathrm{~b}$ gives an example of such order directives.

An order directive is coherent if and only if it ensures the correct computation of the functions it contains. YAO has an internal procedure which verifies the coherence, with respect to the RDG, of the order directives. In the following, we thus assume that all the order directives specified by the user are coherent.

The different order directives are executed in a sequential way in the scheduling specified by the user. The outermost loop (ascendant or descendant) of one order directive corresponds to one axis; in the remainder we use $l$ to denote this axis and $r$ the number of basic functions contained in this outermost loop. As explained in [4], if $l$ is ascendant necessarily all the distances $d_{l}$ of the edges between the $r$ modules are negative or null due to the coherence hypothesis. Symmetrically if $l$ is descendant all the distances $d_{l}$ are positive or null.

\footnotetext{
${ }^{2} \mathrm{~A}$ directed multigraph is a graph with multiple parallel edges.

${ }^{3}$ As for the analogy between the EDG and the modular graph, the RDG has one statement per node while the YAO RDG has a basic function (a set of statements) per node.
} 


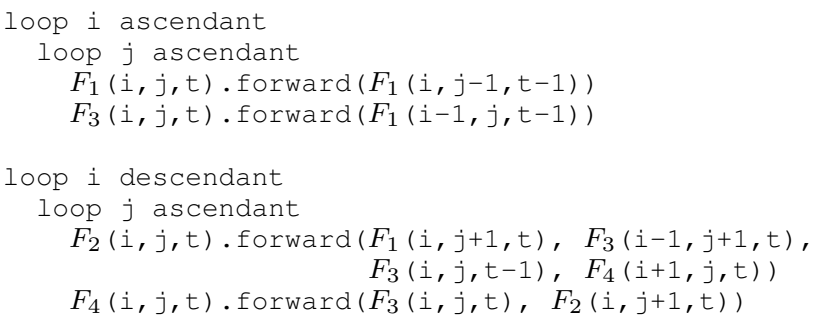

Fig. 3. YAO generator translation of the directives of Figs. 1a and $1 b$.

2) Generation of the forward and backward procedures: in Fig. 3 we give the translation, performed by the YAO code generator, of the ctin and order directives given in Figs. 1a and 1b. This represents the translation, in a pseudo code language, of the forward procedure. Each order directive generates one loop, one for each dimension of the space. The way we traverse the axes, ascendant or descendant, and the scheduling of the modules are the ones specified in the order directives. For each object of each module class $F_{p}$ the local forward function (a $\mathrm{C}++$ method) is called with the output of its predecessor modules as inputs. The local forward functions are defined, for each basic function, by the user. It has to be noticed that all forward functions are thread-safe because they compute the result with respect to the generic grid point $I$, as shown in Fig. 3. The nested loops allow us to compute the output of the modules for all the grid points and for one time step. An overall loop, not shown in the figure, which allows us to traverse the time steps in an incremental order $t, t+1, t+2$, etc., encompasses all the local forward functions. The time loop may be considered as a computation barrier where at current time $t$, all the computations for time $t^{\prime}<t$ are done.

As presented in section II-A2, the backward procedure traverses the modular graph in a reverse topological order. For ease of presentation we do not detail the pseudo code of the backward procedure as we did for the forward procedure. These are very similar. However it is important to point out the addition (accumulation) in the back propagation, explained in [3], [4] and specific to the backward procedure. This accumulation results in output dependencies which may arise between two time steps. This computation is briefly explained in Fig. 4. The $y_{p}$ variables $(p \in 1,2,3)$ are the outputs of the local forward functions. The propagation allows us to provide the predecessor module computations to the successor modules. On the other side, the back propagation allows us to back propagate the gradient of $J$ by using the Jacobian matrix ( $J_{p}$ in the figure) for computing $d x_{p}$. The back propagation of several $d x_{p}\left(d x_{3}\right.$ and $d x_{2}$ in Fig. 4) which have the same predecessor enforces the addition of the $d x_{p}$ (the symbol $\sum$ in the figure).

\section{Algorithm FOR AUtOMATIC PARALLELIZATION}

\section{A. Parallelization of the forward procedure}

In section II we have noted an interesting similarity between the YAO formalism and the theories of compilation and of automatic parallelization of nested loops [10]. Thanks to this

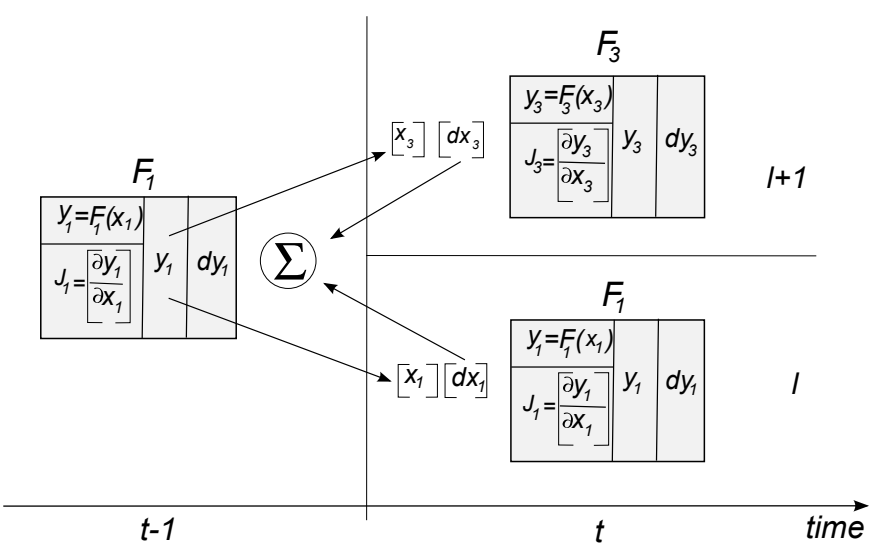

Fig. 4. Addition, represented by the symbol $\sum$, in the back propagation of the backward procedure. These two connections represent a data transfer between two time steps. The two modules $F_{3}$ and $F_{1}$ perform the transfer towards $F_{1}$ at time step $t-1$. This partial graph example case is given by the YAO directives shown in Fig. 1.

similarity we can adapt these techniques and algorithms to the YAO automatic code generator. We thus propose here to integrate and adapt such algorithms in order to automatically parallelize the forward procedure generated by YAO on shared memory architectures with multi-thread programming. No communication is required and we just have to maximize the number of parallel loops. Because of the strong time dependencies in all data assimilation applications the temporal loop is not parallelized and we focus on data parallelism at each time step. The domain decomposition between threads is performed as a 1D block distribution on the space and we rely on a static load balancing since in all the current YAO applications the computation load of each module is constant for each grid point. Our goal is thus to label, as "parallel" or "not parallel", each outermost order directive so that the corresponding loop can be generated as parallel or sequential in the final code (with OpenMP directives). In order to maintain the coherence hypothesis of one given nest of order directives we opted not to change or invert the order defined by the user. However we can still use techniques such as a loop distributions possibly followed by loop fusions in order to detect the maximum available parallelism and to reduce the synchronization points.

Since the temporal loop is not considered in the parallelization algorithm, the edges whose $t^{\prime}-t$ are negative can be removed from the RDG. The remaining graph is shown in Fig. 5, we denote it $\overline{\mathrm{RDG}}$. This is obtained by removing all $d t=-1$ connections and writing only the signs of the distance vector components. Thus $(0,+)$ means a distance vector equal to $(0,+1)$.

Considering some nested order directives which have as outermost axis $l$ and a connection from $F_{s}$ to $F_{d}$ we consider a connection as critical with respect to these nested order directives if:

- $F_{s}$ and $F_{d}$ are contained by the nested directives,

- $d_{t}=0$ and $d_{l} \neq 0$. 


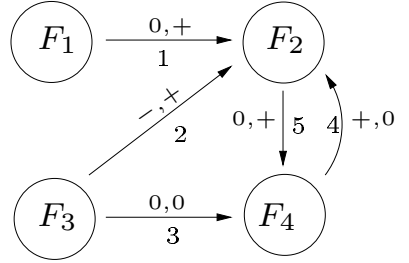

Fig. 5. $\overline{\mathrm{RDG}}$ obtained by simplification of the RDG of Fig. 2. The edges are numbered from 1 to 5 .

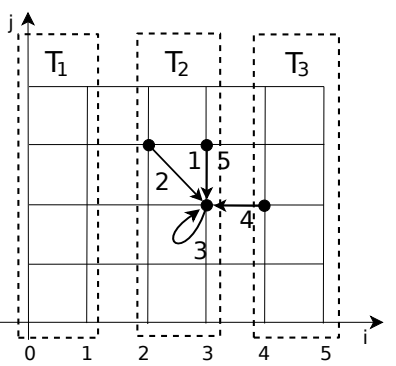
3 threads $T_{1}, T_{2}$ and $T_{3}$. Same edge numbers as in Fig. 5.
Fig. 6. Flow dependencies between

The analysis of the $\overline{\mathrm{RDG}}$ highlights the critical connections which prevents parallelization because of flow dependencies between threads, as presented in Fig. 6. The connection from $F_{4}$ to $F_{2}$ and from $F_{3}$ to $F_{2}$ (edges \#2 and \#4 in Figs. 5 and 6) results in a flow dependency between the couples of threads $\left(\mathrm{T}_{1}, \mathrm{~T}_{2}\right)$ and $\left(\mathrm{T}_{2}, \mathrm{~T}_{3}\right)$ because $d_{l} \neq 0$ (in this example $l$ is the $i$ axis). This is not the case for the connections \#1, 3 and 5 because the two corresponding grid points belong to the domain computed by one thread, as shown in Fig. 6. The connection \#2 is not critical, since $F_{3}$ and $F_{2}$ are not in the same nested loops (see Figs. 1b and 3). In this example only connection \#4 is critical.

For the analysis of one outermost loop $l$ composed by functions $F_{1}, F_{2}, \ldots, F_{r}$ we consider the subgraph $G_{l}$ of the $\overline{\mathrm{RDG}}$ limited to the $r$ basic functions and the edges between them. On the edges of this subgraph we retain only information concerning the distance $d_{l}{ }^{4}$. As far as the $d_{l}$ value is concerned we retain only the sign of $d_{l},(-,+)$ if $d_{l} \neq 0$ and 0 if $d_{l}=0$. The analysis of $G_{l}$ allows to decompose the loop in several loops preserving the computation coherence hypothesis. Taking into account that the forward functions are thread-safe, we can apply the Allen-Kennedy algorithm [12] to decompose the loop in parallel loops as follows.

- Calculate the Strongly Connected Components (SCCs) of $G_{l}$.

- Consider the reduced Directed Acyclic Graph (DAG), denoted by $G_{l / S C C}$, by shrinking each SCC down to a single vertex and by drawing one, and only one, edge between two SCCs if there is at least one edge from the first to the second in the graph $G_{l}$. If at least one of the edge in $G_{l}$ which connects these two SCCs is labeled by non 0 (that is to say either - or + ), then label the corresponding edge in $G_{l / S C C}$ by this value. Else, if all the labels are 0 , then label the corresponding edge in $G_{l / S C C}$ by 0 .

- Sort in a topological order the $G_{l / S C C}$ graph and enumerate all the SCCs following this order. For each SCC generate an $l$ loop which computes its basic functions.

This decomposition is a maximum loop distribution of the

\footnotetext{
${ }^{4}$ All the distances $d_{l}$ in $G_{l}$ are either $\leq 0$ or $\geq 0$ if the $l$ loop is respectively ascendant or descendant, as they correspond to the same outermost loop.
}

initial loop, in other words we can not further decompose without breaking the coherence hypothesis.

We can analyze each SCC loop in order to see if we can perform a domain decomposition on the $l$ axis. For a particular SCC we consider all the edges of the graph $G_{l}$ between two basic functions being part of this SCC. If at least one of these connections is labeled by + or - , namely if $d_{l} \neq 0$, the SCC is considered to be not parallelizable. The loop is parallelizable if all these edges are labeled by 0 . In other words it is parallelizable if it does not contain any flow dependency between threads. We label by $p$ and $\bar{p}$ the loops which are respectively parallelizable and not parallelizable. Such maximum loop distribution gives the largest number of parallel loops. The critical connections of the $\overline{\mathrm{RDG}}$ have been minimized.

\section{B. Reducing synchronization points}

The previous section applies the Allen-Kennedy algorithm to YAO thanks to the analogies between the EDG and the modular graph. This algorithm allows us to automatically label as parallel or not the SCCs resulting in maximum loop distribution. As far as performance is concerned, this loop distribution is not the best solution because it increases the number of synchronization points. Following KennedyMcKinley [11] it is possible to propose a loop fusion algorithm that will reduce the number of synchronization points. As the $G_{l / S C C}$ is a DAG, we can reorganize the SCCs in levels. The levels are numbered from $k=1$ to $k=M_{\text {level }}$ where $M_{\text {level }}$ is the maximum number of levels. The first level, $k=1$, contains the SCCs without predecessors; the predecessors of a SCC at level $k$, with $k>1$, are located in the preceding levels $k^{\prime}, k^{\prime} \leq k-1$, with at least one predecessor located at level $k-1$. Thanks to the level reorganization there are no edges between two vertices at the same level. For each level it is then possible to merge all vertices labeled as $p$ and separately all vertices labeled as $\bar{p}$. We obtain a reduced graph with the same number of levels but with one or two vertices per level. If a level contains two vertices they are mandatory labeled as $p$ and $\bar{p}$.

The fusion process can be extended to the vertices located at two consecutive levels as follows: for all levels $k$ and $k+1$

- merge two vertices labeled as $\bar{p}$, this gives a new $\bar{p}$ vertex;

- merge two vertices labeled as $p$ which are not connected by a critical edge $\left(d_{l}=0\right)$, this gives a new $p$ vertex.

The fusion process between different levels may modify the vertex level repartition. However the modification can affect only some levels: it does not impact the levels which precede $k$. Algorithm 1 allows to manage the fusion of the vertices with the level technique which maintains the highest degree of parallelization. The final reduced graph is treated by YAO which generates code according to the following steps.

- Sort in a topological order the final reduced graph and enumerate all its vertices following this topological order.

- Write one block of order directives for each vertex. These order directives have the same axes as the one provided 


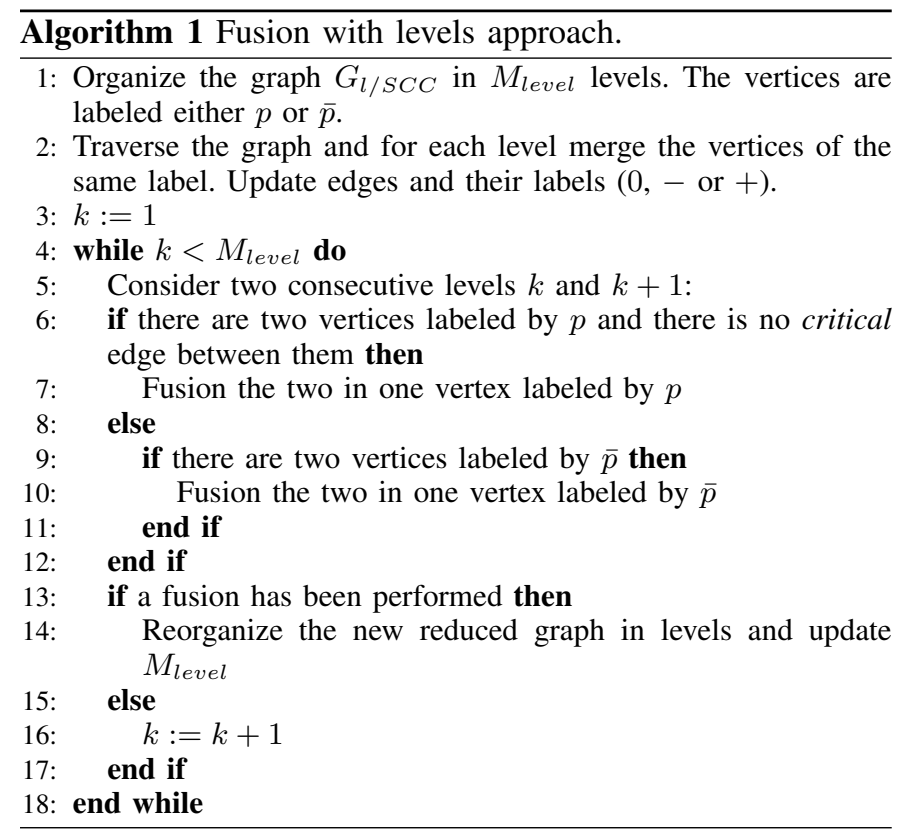

by the user and contain the basic functions merged in the vertex.

\section{Parallelization of the backward procedure}

The same algorithm can also be applied to the backward procedure, which results in a complete parallelization of all computations at each time step. The total elapsed time in a YAO application is mainly composed by the forward and the backward elapsed times. Making parallel these two procedures means that most of the application has been optimized. Profiling measurement on some YAO applications showed that roughly 99 percent of the total elapsed time is, in general, in these procedures.

The RDG used for the backward procedure is the same as for the forward procedure but the arrows are reversed with respect to the original RDG. These two RDGs have the same SCCs. As the outermost loops also have the same axis, the same method used to parallelize the forward procedure is also valid to parallelize the backward procedure. Likewise, it is easy to see that the rules used to merge loop blocks previously introduced remain valid for the backward procedure. Thus, parallel order directives obtained by the decomposition/merging methods defined for the forward procedure can be fully retained for the backward procedure.

However the parallelization of the backward procedure has a further difficulty in terms of synchronization. This synchronization is enforced by the addition (accumulation) presented in II-B2. As shown in Fig. 4, in a parallel context this addition may result in a data race condition (write/write conflicts) if the back propagations of $d x_{p}$ are performed concurrently by several threads. This kind of synchronization may arise between two time steps. Hence, the analysis of the $\overline{\mathrm{RDG}}$ is not sufficient to point out all the data race conditions of the backward procedure.

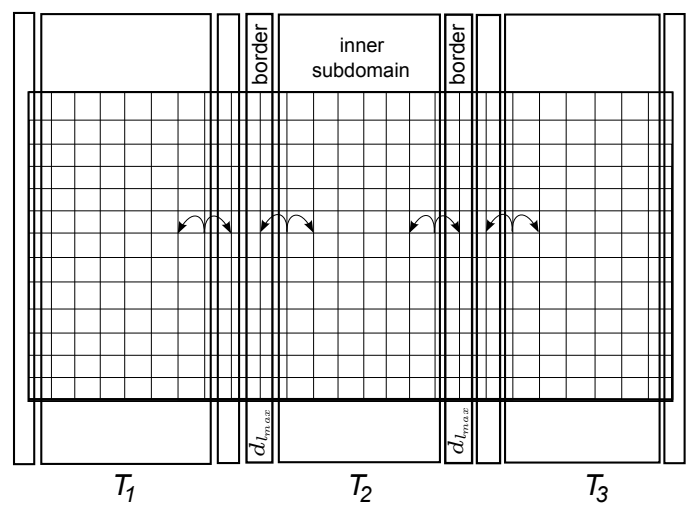

Fig. 7. Subdomain decomposition with $d_{l_{\max }}=1$ for threads $T_{1}, T_{2}$ and $T_{3}$. Each thread domain is decomposed into two border subdomains with $d_{l_{\max }}$ grid points in the parallel dimension, and one inner subdomain.

This can be solved with OpenMP atomic directives which ensure that each addition is performed atomically. However these atomic instructions are costly, as well as numerous in the backward parallel code, which prevents us from obtaining good parallel performances in practice. In order to avoid these OpenMP atomic directives, we rely on the distance vectors of the RDG to determine the maximum $\left|d_{l}\right|$, denoted $d_{l_{\max }}$. In the 1D block decomposition, we can now further decompose each thread domain in three subdomains: two border subdomains with $d_{l_{\max }}$ grid points in the parallel dimension, and one inner subdomain with usually much more than $d_{l_{\max }}$ grid points in the parallel dimension. An example with $d_{l_{\max }}=1$ is presented in Fig. 7.

Data race conditions are now avoided by ensuring that all threads compute the three subdomains in the same ordering. OpenMP barrier directives are required between each subdomain computation. If there are too many threads, or if the computational space is too small, the thread domains may be too small: in these special cases the parallel code generated by YAO will have to rely on OpenMP atomic directives to run correctly in parallel without any data race condition.

Taking all this into account, the overall parallelization algorithm ensures the parallelization of all the computations done by a YAO generated application. It gives a domain decomposition with respect to the outermost loop $l$, which can then be automatically parallelized in the final generated code thanks to OpenMP directives. Furthermore if a multilevel parallelization is desired, it is then possible to apply the same algorithm for each subloop. We emphasize that the parallel code generated by YAO respects the order and the ctin directives which implies that the result of the parallel code is the same as the sequential code.

\section{Marine acoustics example}

This section presents an example of the decomposition algorithm on a 2D modular graph taken from one of the actual YAO applications. The Marine acoustics example has a small number of functions $F_{i}$, which allows us to easily show the evolution of the RDG graph. We use the same function names 


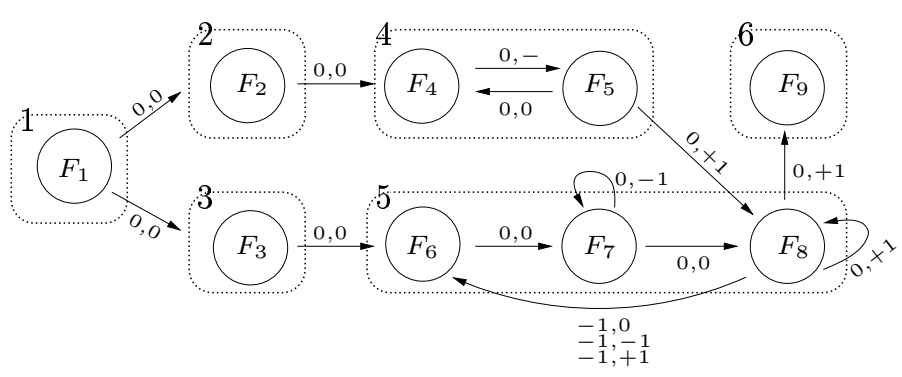

Fig. 8. RDG: the dashed lines are the Strongly Connected Components.

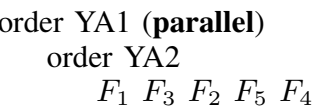

order YA1 order YA2

$\begin{array}{lllllllllllll}F_{1} & F_{3} & F_{2} & F_{5} & F_{4} & F_{6} & F_{7}\end{array}$ order YB2 $F_{8} F_{9}$

\author{
order YA1 (non parallel) \\ order YA2$$
F_{6} F_{7}
$$$$
\text { order YB2 }
$$$$
F_{8}
$$ \\ order YA1 (parallel) \\ order YA2 \\ $F_{9}$
}

Fig. 9. order directives defined by the user for the Marine acoustics example.
Fig. 10. order directives recomputed by the algorithm for the Marine acoustics example.

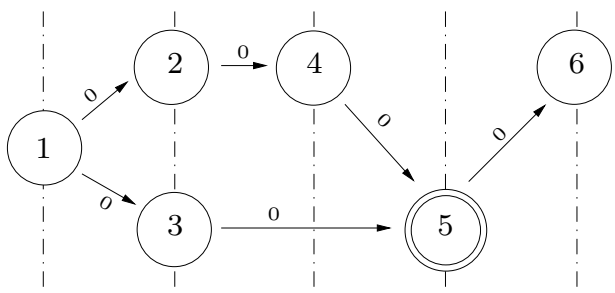

Fig. 11. $G_{l / S C C}$ where the double circle represents a non parallelizable vertex.

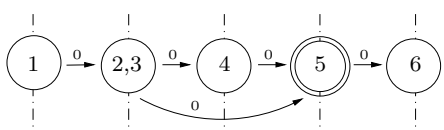

Fig. 12. Fusion of the vertices 2 and 3 in a new $p$ vertex called 2,3.

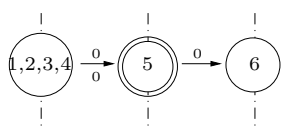

Fig. 13. Fusion of the vertices $1,2,3$ and 4 in a new $p$ vertex called $1,2,3,4$.

reached $M_{\text {level }}$ equals 3 . This topological order is translated in an ordering of the modules. The final scheduling respects the ordering given by the user and corresponds to: $\left[\begin{array}{ll}F_{1} & F_{3}\end{array}\right.$ $\left.F_{2} \quad F_{5} \quad F_{4}\right],\left[\begin{array}{lll}F_{6} & F_{7} & F_{8}\end{array}\right],\left[F_{9}\right]$ or [n(z) B C gam bet], [R $X_{t}$ $\psi],\left[\psi_{f d}\right]$. The final order directive decomposition is given in Fig. 10. With the keywords parallel and non parallel the figure outlines the outermost loops (order directives) that the algorithm has recognised as parallel or not.

\section{Performance Results}

as [6], [5]. This YAO application deals with marine acoustics and allows to assimilate some actual observations of acoustic pressure in order to retrieve some geoacoustic parameters like celerity, density and attenuation. In [6] the basic functions are denoted by $n(z), C, B$, bet, gam, $R, X_{t}, \psi$ and $\psi_{f d}$. To make it simpler we denote them respectively by $F_{1}, \ldots, F_{9}$. Fig. 8 shows the $R D G$ composed by $r=9$ basic functions and the edges labeled with the coefficient signs of the ctin directives. In this figure the SCCs are outlined by the dashed lines and numbered from 1 to 6 . The order directives specified by the user are given in Fig. 9. In this case, the outermost loop is related to the ascendant $i$ axis. After computing the $G_{l / S C C}$ graph, we label each vertex and we proceed with the level reorganization, as presented in Fig. 11, where $M_{\text {level }}$ equals 5 ; the single circle is a parallelizable vertex $(p)$ and the double circle is a non parallelizable vertex $(\bar{p})$. Fig. 12 shows the fusion of the vertices 2 and 3 labeled by $p$ in a new vertex called 2,3 of the same label. This is done in the initialization phase of the algorithm (step 2). Then the vertices 1 and 2,3 can be merged in a new vertex called 1,2,3 which is parallel too. The two vertices are located on levels $k=1$ and $k=2$. A level reorganization reduces $M_{\text {level }}$ to 4 . The same operation is done on the modules 1,2,3 and 4, followed again by a level reorganization ( $M_{\text {level }}$ reduced to 3 ). The topological order is then: [1,2,3,4], [5], [6] as shown in Fig. 13. The algorithm ends because it is no longer able to fusion and the level counter has

We now present the performance results of the parallel code generated by YAO for two actual applications of data assimilation: the Shallow-water and the Marine acoustics applications mentioned before. These tests have been performed on a server, located at Polytech Paris-UPMC (Paris, France), composed of one AMD Magny-Cours Opteron 6168 processor and $16 \mathrm{~GB}$ of memory. This processor has 12 cores running at $1.9 \mathrm{GHz}$ which have private $\mathrm{L} 1 / \mathrm{L} 2(64 \mathrm{~KB} / 512 \mathrm{~KB})$ caches and share two 6MB L3 caches. All computations are performed in double precision.

The RDG of the Shallow-water application is composed by $6 \mathrm{SCC}$ (each SCC contains one basic function), see [3] for more details. The parallelization algorithm returns that all SCCs are parallelizable. Fig. 14 shows the elapsed time and the parallel speedup for an increasing number of cores used (with one OpenMP thread per core) and for different computational space sizes, with both OpenMP atomic directives and our subdomain decomposition. The data race conditions in the backward procedure are more efficiently avoided with our subdomain decomposition which clearly offers better performance than the atomic directives. We emphasize that such OpenMP code automatically generated by YAO is equivalent to a (nontrivial) manual parallelization, and offers good speedups (up to 9.4 on 12 cores). Moreover for a fixed number of cores the speedup increases with the computational space size since this increases the computational granularity of each thread.

The performance results on the Marine acoustics are very 

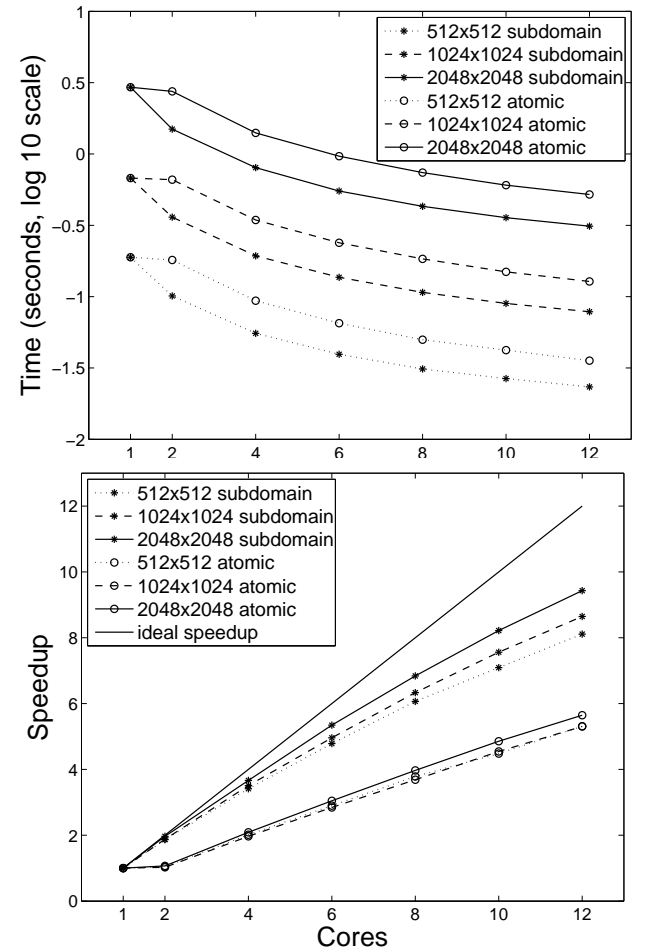

Fig. 14. Shallow-water performance measurements for three 2D computational space sizes over one time step (time averaged over 30 time steps). Both performances with OpenMP atomic directives and subdomain optimization are shown. The time encompasses both the forward and the backward procedures.

different. In section III-D we have shown that the parallelization algorithm does not parallelize the whole GDR. Three modules, which unfortunately contain most of the computation, are excluded from the parallel region. Fig. 15 shows the elapsed time and the parallel speedup, as well as the theoretical maximum speedup according to Amdahl's law for this application. The parallel speedup is very limited, but the code generated by YAO offers most of the speedup available for this application. Again, the performance gain increases with the computational space size.

\section{CONCLUSION AND PERSPECTIVES}

In this paper, we have shown how the modular graph formalism of YAO allows addressing the issue of automatic parallelization of the code generated by YAO. Indeed, the YAO modular graph is generated by a reduced graph, which is similar to the Reduced Dependence Graph (RDG) used in automatic parallelization of nested loops. This similarity allows the adaptation to YAO of the algorithms that were developed in this research field. We have thus presented here how the Allen-Kennedy [12] and Kennedy-McKinley [11] algorithms can be integrated and adapted in order to enable the automatic parallelization, via multiple threads on shared memory architectures, of the application code generated by YAO. In the backward procedure the modular graph is furthermore used to decompose each thread domain into three subdomains, whose appropriate sizes enable us to completely avoid the race
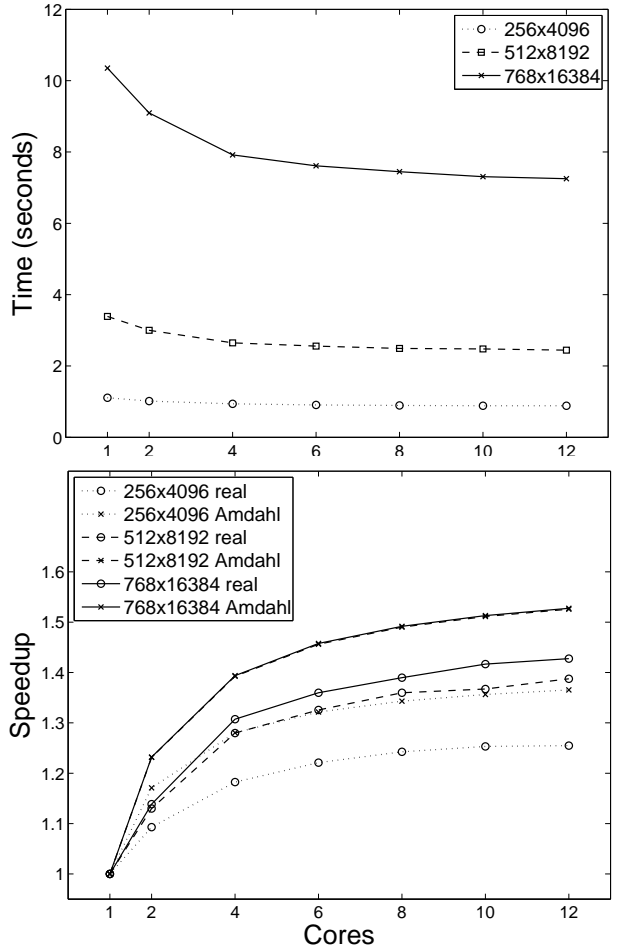

Fig. 15. Marine acoustics performance measurements for three 2D computational space sizes over one time step (time averaged over 30 time steps). The time encompasses both the forward and the backward procedures.

conditions of this backward procedure. We have also presented the performance results of the parallel generated code with OpenMP on a multicore CPU for two actual applications: the Shallow-water and the Marine acoustics. We obtain good speedups (for example up to 9.4 on 12 cores) within the limits of the parallelism available in the application.

More advanced transformations (unimodular transformation, loop inversion, SIMD vectorization, tiling, ...) have already been developed in automatic loop parallelization, especially via the polyhedral model [10], [15]. We are currently studying if and how this polyhedral model can be integrated in the YAO framework. In the future, we also plan to investigate the automatic generation of MPI code from OpenMP code in the YAO context in order to automatically scale data assimilation applications on distributed memory architectures. It can be noticed that the subdomain decomposition between border and inner subdomains, presented here to avoid race conditions, may help overlap MPI communications with computation in order to obtain the best speedups in this distributed memory context: here again, the modular graph of YAO may be very useful to automatically determine this subdomain decomposition for any variational data assimilation application.

\section{ACKNOWLEDGMENT}

The authors acknowledge funding from Emergence-UPMC2010 research program. 


\section{REFERENCES}

[1] E. Kalnay, Atmospheric Modeling, Data Assimilation, and Predictability. Cambridge University Press, 2003.

[2] O. Talagrand, "Assimilation of Observations, an Introduction," J. Meteor Soc. Japan, vol. 75, pp. 191-209, 1997.

[3] L. Nardi, C. Sorror, F. Badran, and S. Thiria, "YAO: A Software for Variational Data Assimilation Using Numerical Models." in LNCS 5593, Computational Science and Its Applications - ICCSA 2009, pp. 621-636.

[4] L. Nardi, "Formalisation et automatisation de YAO, générateur de code pour l'assimilation variationnelle de donnèes," $\mathrm{PhD}, \mathrm{CNAM}, 2011$.

[5] F. Badran, M. Berrada, J. Brajard, M. Crépon, C. Sorror, S. Thiria, J.-P. Hermand, M. Meyer, L. Perichon, and M. Asch, "Inversion of Satellite Ocean Colour Imagery and Geoacoustic Characterization of Seabed Properties: Variational Data Inversion Using a Semi-automatic Adjoint Approach," J. of Marine Systems, vol. 69, pp. 126-136, 2008.

[6] M. Berrada, "Une approche variationnelle de l'inversion, de la recherche locale à la recherche globale par carte topologique: application en inversion géoacoustique," PhD Thesis, UPMC, France, 2008.

[7] J. Brajard, C. Jamet, C. Moulin, and S. Thiria, "Use of a Neurovariational Inversion for Retrieving Oceanic and Atmospheric Constituents from Satellite Ocean Colour Sensor: Application to Absorbing Aerosols," Neural Networks, vol. 19, no. 2, pp. 178-185, 2006.

[8] A. Kane, S. Thiria, and C. Moulin, "Développement d'une Méthode d'Assimilation de Données in Situ dans une Version 1D du Modèle de Biogochimie Marine PISCES," Master's thesis, LSCE/IPSL, CEACNRS-UVSQ, 2006.

[9] G. Madec, NEMO ocean engine. LOCEAN, Paris, France: Note du Pôle de modélisation de l'Institut Pierre-Simon Laplace No 27, 2008.

[10] A. Darte, Y. Robert, and F. Vivien, Scheduling and automatic parallelization, Birkhaüser, Ed., 2000

[11] K. Kennedy and K. McKinley, "Typed fusion with applications to parallel and sequential code generation," Tech. Rep., 1993.

[12] R. Allen, D. Callahan, and K. Kennedy, "Automatic decomposition of scientific programs for parallel execution," in POPL '87: Proceedings of the 14th ACM SIGACT-SIGPLAN symposium on Principles of programming languages. NY, USA: ACM, 1987, pp. 63-76.

[13] H. Jin, M. A. Frumkin, and J. Yan, "Automatic Generation of OpenMP Directives and Its Application to Computational Fluid Dynamics Codes," in ISHPC 2000. Springer-Verlag, pp. 440-456.

[14] J. Taillard, F. Guyomarc'h, and J.-L. Dekeyser, "A Graphical Framework for High Performance Computing Using An MDE Approach," in Proceedings of the 16th Euromicro Conference on Parallel, Distributed and Network-Based Processing, PDP 2008. USA: IEEE CS, pp. 165-173.

[15] U. Bondhugula, A. Hartono, J. Ramanujam, and P. Sadayappan, "A practical automatic polyhedral parallelizer and locality optimizer," in PLDI 2008. USA: ACM SIGPLAN, pp. 101-113. 